\title{
Scintillation detectors for fast neutrons
}

\section{Horst Klein *}

Physikalisch-Technische Bundesanstalt (PTB)

Bundesallee 100, 38116 Braunschweig, Germany

E-mail: horst.klein@ptb.de

\section{Frank D. Brooks}

Department of Physics

University of Cape Town

Rondebosch 7700, South Africa

E-mail: fbrooks@science.uct.ac.za

\begin{abstract}
The properties and various applications of scintillation detectors for neutrons of energy $>1 \mathrm{keV}$ are reviewed. Characteristics of the organic scintillator neutron detectors are discussed in some detail and the energy resolution achieved in neutron spectrometric applications is reviewed. Present trends and future needs in neutron detection are summarised and discussed.
\end{abstract}

International Workshop on Fast Neutron Detectors

University of Cape Town, South Africa

April 3-6, 2006

\footnotetext{
${ }^{*}$ Speaker
} 


\section{Introduction}

For the purposes of FNDA-2006 a neutron of kinetic energy that exceeds $1 \mathrm{keV}$ is regarded as "fast". In this review we shall relate to a wide range of applications of scintillation detection of fast neutrons and particularly to the following: "straightforward" detection (counting) of fast neutrons; detection with timing, for example for coincidence measurements or neutron time-of-flight measurements; detection for neutron spectrometry based on measurements of either time-of-flight spectra or pulse height spectra; detection for the accurate measurement of neutron fluence; detection for radiation protection dosimetry; and detection for practical applications of neutron technology in other fields, for example, neutron radiography, contraband detection and plasma diagnostic measurements.

The classic reference (and compulsory reading!) for this topic is the review article "Scintillation detectors for neutron physics research" contributed by Harvey and Hill [1] to the special issue (1979) of Nuclear Instruments and Methods, "Detectors in Nuclear Science", edited by D.A. Bromley [2]. Other articles in this volume deal with the related topics of neutron time-of-flight spectrometers [3] and organic scintillators [4]. The basics of neutron detection and scintillation counting are covered in the volumes "Fast Neutron Physics, Parts 1 and 2", edited by J.B. Marion and J.L. Fowler [5] and in textbooks on radiation detection [6,7] and scintillation counting [8]. Some recent developments are presented in other review articles [913].

In this review we consider three topics. First, we provide a brief summary of the types of scintillation detector that we consider most useful in fast neutron work today. Second, we summarise and discuss the most relevant characteristics of these detectors. And third, we attempt to identify present and future trends in the development and application of scintillation detectors for fast neutrons.

\section{Summary of scintillation detectors for fast neutrons}

For convenience we define three energy regions as follows, in order to make comparisons between neutron detectors: the "low-energy" region, 1-300 keV; the "medium-energy" region, 0.1-20 MeV; and the "high-energy" region, > $20 \mathrm{MeV}$. To compare different detectors we present a table for each energy region in which the following data are listed: the nuclear mode on which detection is based, for example elastic scattering $(n, n)$ or nuclear reaction $(n, x)$ leading to emission of charged particle(s); the capability of the detector for determining neutron energy spectra from pulse height spectra measurements, indicated under the heading "PHS"; typical timing resolution achieved $(\Delta t)$; pulse shape discrimination capability for effective discrimination against gamma-rays, indicated under the heading "PSD"; and references to original and recent publications. PHS and PSD capabilities are indicated by either a "yes", a "no" or (if unknown) a "?". 


\subsection{Low-energy neutron detectors}

The characteristics of selected detectors for the low-energy range, 1-300 keV, are presented in Table 1. Neutron research in this energy region was more active prior to 1979 than it is now. Neutron detection developments prior to1979 are reviewed in refs. [1,5]. Notable new developments, subsequent to 1979, are the boron-loaded plastic scintillator [14] (detector 3 in Table 1) and the lithium-loaded liquid scintillator [15-18] (detector 6). ]. The ${ }^{6} \mathrm{Li}$-loaded glass scintillator (detector 5) is well-suited for application over the whole of this energy range. The organic scintillators (detectors 7-9) are best-suited for use at the high-energy end of the range $(>0.1 \mathrm{MeV})$.

\begin{tabular}{|c|l|c|c|c|c|l|}
\hline No. & Type & Mode & PHS & $\Delta t(\mathrm{~ns})$ & PSD & References \\
\hline 1 & ${ }^{10} \mathrm{~B}-\mathrm{plug}+$ NaI & $(\mathrm{n}, \alpha)$ & no & 10 & no & 19 \\
\hline 2 & ${ }^{10} \mathrm{~B}-\mathrm{CH}$ liquid & $(\mathrm{n}, \alpha)$ & no & 400 & yes & $5,20-27$ \\
\hline 3 & ${ }^{10} \mathrm{~B}-\mathrm{CH}$ plastic & $(\mathrm{n}, \alpha)$ & no & 400 & no & $14,28-31$ \\
\hline 4 & ${ }^{6} \mathrm{LiI}(\mathrm{Eu})$ crystal & $(\mathrm{n}, \alpha)$ & no & 200 & no & 5,32 \\
\hline 5 & ${ }^{6} \mathrm{Li}$-glass & $(\mathrm{n}, \alpha)$ & no & 5 & no & $33-40$ \\
\hline 6 & ${ }^{6} \mathrm{Li}-\mathrm{CH}$ liquid & $(\mathrm{n}, \alpha)$ & no & 400 & yes & $15-18$ \\
\hline 7 & $\mathrm{CH}(\mathrm{D})$ crystal & $(\mathrm{n}, \mathrm{n})$ & yes & 2 & yes & 1,41 \\
\hline 8 & $\mathrm{CH}(\mathrm{D})$ liquid & $(\mathrm{n}, \mathrm{n})$ & yes & 2 & yes & $1,42,43$ \\
\hline 9 & $\mathrm{CH}(\mathrm{D})$ plastic & $(\mathrm{n}, \mathrm{n})$ & yes & 1 & no & 1 \\
\hline
\end{tabular}

Table 1: Scintillation detectors for $E_{n}=1-300 \mathrm{keV}$

The timing resolutions quoted for boron-loaded and lithium-loaded organic scintillators (detectors 2, 3 and 6 in Table 1) are those applying for the detection of low-energy neutrons $(<100 \mathrm{keV})$. These values are relatively poor (large) due to the neutron energy-moderation process which precedes neutron capture in ${ }^{10} \mathrm{~B}$ or ${ }^{6} \mathrm{Li}$. Proton recoils produced in this process (by low-energy neutrons) generate a signal that is usually below the detection threshold. In the case of higher energy incident neutrons the recoil proton signal may be detected in addition to, and ahead of, the neutron capture pulse. If so, then a much better timing resolution may be obtained, based on the initial recoil proton pulse. In this case the characteristic double-pulse signature [20] is also useful for identifying the neutron event. The main applications of boronloaded and lithium loaded organic scintillators today are those that make use of the double-pulse signature feature.

\subsection{Medium-energy neutron detectors}

Characteristics of detectors for the medium-energy range $(0.1-20 \mathrm{MeV})$ are presented in Table 2. The main workhorses in this group are the organic crystals, liquids and plastics (detectors 3-5). The recently described [44,45] organic gel scintillator (detector 6), promises to be particularly useful, firstly because it is reported to have performance characteristics similar to those of the well-known liquid scintillators, NE213, BC501A and EJ305, and secondly (and 
importantly) because it is not considered a fire hazard, as is the case for the liquid scintillators. The liquid-helium and liquid-xenon detectors (7-9) were developed for specific applications, for example, for incorporation in a neutron polarization analyser in the case of detector 8 .

\begin{tabular}{|c|l|c|c|c|c|l|}
\hline No. & Type & Mode & PHS & $\Delta t(\mathrm{~ns})$ & PSD & References \\
\hline 1 & ${ }^{6}$ LiI(Eu) crystal & $(\mathrm{n}, \mathrm{\alpha})$ & yes & 200 & no & 5,32 \\
\hline 2 & ${ }^{6}$ Li-glass & $(\mathrm{n}, \mathrm{\alpha})$ & no & 5 & no & $33-40$ \\
\hline 3 & $\mathrm{CH}(\mathrm{D})$ crystal & $(\mathrm{n}, \mathrm{n}),(\mathrm{n}, \mathrm{x})$ & yes & 2 & yes & $1,46-49$ \\
\hline 4 & $\mathrm{CH}(\mathrm{D})$ liquid & $(\mathrm{n}, \mathrm{n}),(\mathrm{n}, \mathrm{x})$ & yes & 2 & yes & $43,50-67$ \\
\hline 5 & $\mathrm{CH}(\mathrm{D})$ plastic & $(\mathrm{n}, \mathrm{n}),(\mathrm{n}, \mathrm{x})$ & yes & 1 & no & $66,68-76$ \\
\hline 6 & $\mathrm{CH}(\mathrm{D})$ gel & $(\mathrm{n}, \mathrm{n}),(\mathrm{n}, \mathrm{x})$ & yes & 2 & yes & 44,45 \\
\hline 7 & Liquid ${ }^{3} \mathrm{He}$ & $(\mathrm{n}, \mathrm{n}),(\mathrm{n}, \mathrm{x})$ & yes & 2 & no & 77,78 \\
\hline 8 & Liquid ${ }^{4} \mathrm{He}$ & $(\mathrm{n}, \mathrm{n})$ & yes & 2 & no & 79 \\
\hline 9 & Liquid Xe & $(\mathrm{n}, \mathrm{x})$ & yes & $?$ & $?$ & 80 \\
\hline 10 & $\mathrm{Cs}(\mathrm{Tl})$ crystal & $(\mathrm{n}, \mathrm{x})$ & yes & $?$ & yes & 81,82 \\
\hline
\end{tabular}

Table 2: Scintillation detectors for $E_{n}=0.1-20 \mathrm{MeV}$

\subsection{High-energy neutron detectors}

Characteristics of detectors for the high-energy range $(>20 \mathrm{MeV})$ are presented in Table 3. The organic scintillators (detectors 1-3) are also the leading members of this group, together with the barium fluoride crystal (detector 5). Three factors that become more important at higher neutron energies should be noted. Firstly, even in the organic scintillators, the role of elastic scattering $(n, n)$ on hydrogen becomes less important than that of the $(n, x)$ reactions on carbon in the detection process, due to the changes in the cross sections for these two processes with increasing neutron energy. Secondly, the cross sections for $(n, x)$ reactions on the nuclides

\begin{tabular}{|c|l|c|c|c|c|l|}
\hline No. & Type & Mode & PHS & $\Delta t(\mathrm{~ns})$ & PSD & References \\
\hline 1 & $\mathrm{CH}(\mathrm{D})$ liquid & $(\mathrm{n}, \mathrm{n}),(\mathrm{n}, \mathrm{x})$ & yes & 2 & yes & $67,83-91$ \\
\hline 2 & $\mathrm{CH}(\mathrm{D})$ plastic & $(\mathrm{n}, \mathrm{n}),(\mathrm{n}, \mathrm{x})$ & yes & 1 & no & $76,92-94$ \\
\hline 3 & $\mathrm{CH}(\mathrm{D})$ gel & $(\mathrm{n}, \mathrm{n}),(\mathrm{n}, \mathrm{x})$ & yes & 2 & yes & 44,45 \\
\hline 4 & Liquid ${ }^{3} \mathrm{He}$ & $(\mathrm{n}, \mathrm{n}),(\mathrm{n}, \mathrm{x})$ & yes & 2 & no & 77,78 \\
\hline 5 & $\mathrm{BaF}_{2}$ crystal & $(\mathrm{n}, \mathrm{x})$ & yes & 1 & yes & $13,82,95-98$ \\
\hline 6 & $\mathrm{BGO}$ crystal & $(\mathrm{n}, \mathrm{x})$ & $?$ & $?$ & $?$ & $13,98,99$ \\
\hline 7 & $\mathrm{CsI}(\mathrm{Tl})$ crystal & $(\mathrm{n}, \mathrm{x})$ & yes & $?$ & yes & $13,81,82$ \\
\hline 8 & $\mathrm{PbWO}_{4}$ crystal & $(\mathrm{n}, \mathrm{x})$ & $?$ & $?$ & $?$ & $13,100-103$ \\
\hline
\end{tabular}

Table 3: Scintillation detectors for $E_{n}>20 \mathrm{MeV}$

of inorganic scintillators like $\mathrm{BaF}_{2}$ are comparable with, or even higher than, those for $(\mathrm{n}, \mathrm{x})$ reactions on ${ }^{12} \mathrm{C}$ at high energies. Thirdly, inorganic crystal materials are typically higher- $Z$ and denser than organic scintillator materials and therefore present more nuclei per unit volume to the incident neutron beam as well as a higher stopping power for the charged nuclear reaction products released in the neutron detection process. The combined effects of these three factors 
are, firstly, to make inorganic scintillators like $\mathrm{BaF}_{2}$ more efficient neutron detectors than organic scintillators at high energy, when compared at the same physical size [95], and secondly to reduce the effects of charged particle escape (wall effect) from the scintillator. With these considerations in mind it seems likely that other inorganic scintillators such as detectors 6-8 in Table 3 may also become popular for high-energy neutron detection in future.

\section{Characteristics of organic scintillation detectors}

Among all scintillation detectors employed for fast neutron measurements, organic scintillation detectors are preferentially used for counting and also for spectrometry, either by the time-of-flight method, if appropriate pulsed or tagged neutron sources are available, or by unfolding of pulse height spectra. All techniques require well characterised detector systems, in particular concerning the non-linear light output for all secondary charged particles and for the scintillation pulse shape.

The properties of scintillation detectors are discussed in detail in textbooks $[5,6,8]$ and reviews [2]. Information on these properties is based on experimental investigations and calculations. Calculations are now based on Monte Carlo (MC) simulations which, in turn, require a sound basis of parameters, specific to the particular detector system, and reliable cross sections for the interaction of the neutrons with the constituents of the detector assembly.

In the case of the organic scintillation detectors the most important properties to be considered are:

- the light output functions for electrons $L_{e}\left(E_{e}\right)$ and ions $L_{p, d, \alpha, . .}\left(E_{i}\right)$,

- the light collection from the scintillator to the phototube,

- the pulse height resolution function $\mathrm{d} L(L) / L$,

- the pulse height response functions and the detection efficiencies for neutrons and gamma rays, as a function of the energy of the incident radiation,

- the time response and resolution,

- the pulse shape discrimination capability and

- the energy resolution in tof-spectrometry and in unfolding methods.

Recent progress in determining these properties will be discussed.

\subsection{Light output of organic scintillators}

The light output (or scintillation pulse height output response) $L$ of different scintillators to ionizing particles of different charge, mass and energy $E$ has been studied since the invention of scintillation detectors. For the organic scintillators it is well-known that the dependence of $L$ on $E$ is generally non-linear and differs for different types of particles. This feature is sometimes regarded as a major disadvantage of organic scintillators, particularly in regard to their application as neutron detectors. Nonetheless, more than fifty years ago Birks [104] showed that the non-linear response of organic scintillators can be modelled in terms of a simple relationship 
between the specific fluorescence (light emission per unit distance along the path of the ion) $\mathrm{d} L / \mathrm{d} x$ and the specific energy loss $\mathrm{d} E / \mathrm{d} x$ of the ion, which increases as the ion slows down. Alternative relationships between these variables were proposed by Chou [105] and Wright [106] and were also successful in explaining the experimental data. Later on a more detailed model that takes account of the spatial distribution (lateral as well as linear) of the primary ionization and also of the kinetics governing the emission of prompt and delayed scintillation components was developed by Voltz et al. [107-111] and found to give a better description (see Ref [4]) of the experimental data for a wider range of energies and types of ions. However, the earlier and simpler formulae [104-106] nevertheless appear to be suitable for characterizing the $L(E)$ dependence of the organic scintillators used for neutron detection and were used, for example, by Craun and Smith [112] to describe the systematic and comprehensive investigations that they made of the light output responses of some plastic, liquid and crystal scintillators to protons and electrons [113].

The response of an organic scintillator to electrons may be determined from the pulse height spectrum of Compton electrons released in the scintillator by monoenergetic gamma rays. A response measurement is obtained by determining the pulse height that corresponds to the known electron energy of the Compton edge observed in the pulse height spectrum. The response to protons may similarly be obtained from the upper limit of the pulse height spectrum of recoil protons produced by elastic n-p scattering of monoenergetic neutrons in the scintillator. The pulse height spectra resulting from such measurements are continuous and correspond to the response functions of the scintillator for gammas and neutrons respectively, of the corresponding incident energy. If sets of such measurements are made for the same scintillator using several different incident gamma and neutron energies then the ratio of the responses to electrons and protons is obtained directly, as a function of incident energy. Response functions for the detector for different incident gamma and neutron energies are also obtained from these measurements and can be compared with response functions calculated from Monte Carlo simulations. Careful investigations of this type were initiated in the nineteen-sixties by Verbinski et al. [114] and established the first complete response matrix for an organic scintillator based on experimental data for mono-energetic neutrons.

Response data and response functions obtained by these methods should be carefully examined, however, before being generally applied, for the following reasons:

- Different recipes have been applied to determine the pulse height that corresponds to the Compton edge in the pulse height distribution for gamma-rays (see Fig. 10 of [115]).

- Various proton light output functions published for NE213 and similar liquid scintillators prior to 1979, exhibited remarkable differences in absolute scale and shape even if they were renormalised to the same reference for electrons (see Fig. 1 of [116]).

- Experimental measurements should include tests to confirm that the pulse height measuring system is free from saturation effects or other sources of non-linearity such as those that can occur in photomultiplier tubes. Unfortunately this confirmation is absent from some of the data reported in the literature. 
- It is important that the photomultiplier output integration time constant used for the pulse height measurement should be long enough to ensure that the fraction of the slow scintillation component that is lost from the measurement does not significantly affect the result. For the well-known crystal and liquid scintillators, for example, this integration constant should be at least $1 \mu \mathrm{s}$. The value of the time constant used in the measurements should be given.

For these reasons the analysing procedure used for reference data must first be standardised. Measured Compton spectra are compared with MC-simulated responses which are properly folded with the resolution function. An iterative analysis then results in a non-linear light output for electrons below $40 \mathrm{keV}$ and beyond 1.6 MeV [115,117-119]. On this basis, perfect agreement of measured and simulated Compton spectra can be achieved, both in shape and absolute scale, for gamma-energies up to $20 \mathrm{MeV}$. In this way, a unique light output function has been established for liquid scintillators varying in shape and volume $[118,119]$. Besides the light output function, the resolution parameters are obtained from the calibration with gamma-sources. The proposed recipe is now generally applied for reference.

In a similar way, the proton light output function is deduced from measured response functions for mono-energetic neutrons by comparison with simulated responses. Also the $L_{p^{-}}$ function then results from an iterative procedure. The $L_{p}$-functions measured at PTB for more than 20 NE213-detectors of different volume exhibit significant differences [53] confirming earlier findings. A general parametrisation as for electrons is therefore not possible for ions. Also the light output functions for $\alpha$-particle which are deduced from the contribution due to the ${ }^{12} \mathrm{C}(\mathrm{n}, \alpha)$-reaction for neutron energies $>8 \mathrm{MeV}$, differ significantly. From these results it is concluded, that the light output functions for secondary ions must be determined for each organic detector system individually, taking also into account the electronics and the data acquisition system and the applied analysing procedure as well.

When well-established light output functions are provided the MC-simulated response functions show almost perfect agreement with experiment, for example for neutron energies up to $10 \mathrm{MeV}$ [120]. However, contributions due to $\alpha$-particles from neutron-interactions with the carbon content of the scintillators which were clearly observed in an experimental calibration [121] are not correctly simulated, chiefly due to imperfect knowledge of the relevant cross sections and reaction dynamics. These discrepancies increase with increasing neutron energies [83] because other poorly-known reaction channels, e.g. $(n, d),(n, p)$ or $(n, x n)$ reactions, also contribute to the detection process.

Since complete and satisfying cross section data cannot be expected to become available in the near future for these reactions and the neutron energy range of interest is increasing to some hundreds of $\mathrm{MeV}$, the conclusion can only be: The response matrix has to be determined experimentally and normalised properly, either with reference to other neutron metrology systems, e.g. recoil proton telescopes, or by fitting these spectra to normalised MC simulations in the region of the pulse height spectra where n-p scattering dominates the response. This technique was already used by Verbinski et al. [114] and later on successfully applied by others 
$[120,122]$. Although not reported in such detail for other organic scintillators like stilbene and anthracene crystals or plastic scintillators, similar problems are to be expected also for these organic scintillators.

\subsection{Light collection and pulse height resolution function $\mathrm{dL}(\mathrm{L}) / \mathrm{L}$}

The pulse height resolution is determined as a side product when simulated responses are fitted at the Compton or recoil proton edge of pulse height spectra taken in mono-energetic gamma- or neutron-fields. In general, the pulse height resolution consists of three components, namely the noise of the electronics (variance $C^{2}$ ), the statistics of the number of photoelectrons $\left(B^{2}\right)$ and the variation of the light collection over the detector volume $\left(A^{2}\right)$ which sum up to $\mathrm{d} L / L=\left\{A^{2}+B^{2} / L+C^{2} / L^{2}\right\}^{1 / 2}$ assuming that the contributions are independent and normally distributed [115]. At least for the position-dependent effect the last assumption is questionable. Photon transport calculations $[123,124]$ show that the probability density distribution of photons reaching the cathode of the photomultiplier strongly depends on the size of the scintillator, the reflectivity at the surface and the coupling of the scintillator to the tube, e.g. through a light guide. Even in case of the "standard sized" cylindrical liquid scintillator, $5.08 \mathrm{~cm}$ in diameter and length, asymmetric distributions are obtained as shown in Fig. 5 of [124] if the scintillator is coupled directly or through a fully coated light guide to the tube (showing an upper tail) or through a polished light guide (lower tail). Obviously, an optimisation can easily be achieved in this case by means of a partially coated light guide, resulting in an almost symmetrical distribution. It should be noted that this simple recipe fails if inhomogeneities of the quantum efficiency of the phototube influence the position-dependent response and for detectors with a length larger than the diameter. In the latter case, an optimisation may be achieved by differential coating of the surface of the detector itself [125]. It should, however, be noted that only small $A$-parameters guarantee reasonable pulse height resolution at high pulse heights resulting in better energy resolution at high energies.

In case that a position-independent light collection cannot be realised, the photon transport must be included in the MC-simulation of the response, taking into account the differential light production along the track of the secondary charged particle. Modern MC-codes allow for this option [125-127].

\subsection{Response matrix and detection efficiency}

Provided the chemical composition, chiefly the H/C-ratio, the physical parameters, i.e. size, attenuation length and reflectivity at the surfaces, and the characteristic functions, i.e. light output and resolution functions, are known, the response functions can be reliably simulated $[128,129]$. However, even when the shape of the response function is perfectly reproduced, the absolute value may require an energy-independent correction of up to $5 \%$ dependent on shape and volume of the scintillator [53]. The relative efficiency can then be reliably, i.e. with uncertainty less than $2 \%$, predicted for any actual threshold and for neutron energies up to about $10 \mathrm{MeV}$. Since the contributions due to reactions with the carbon content are not well 

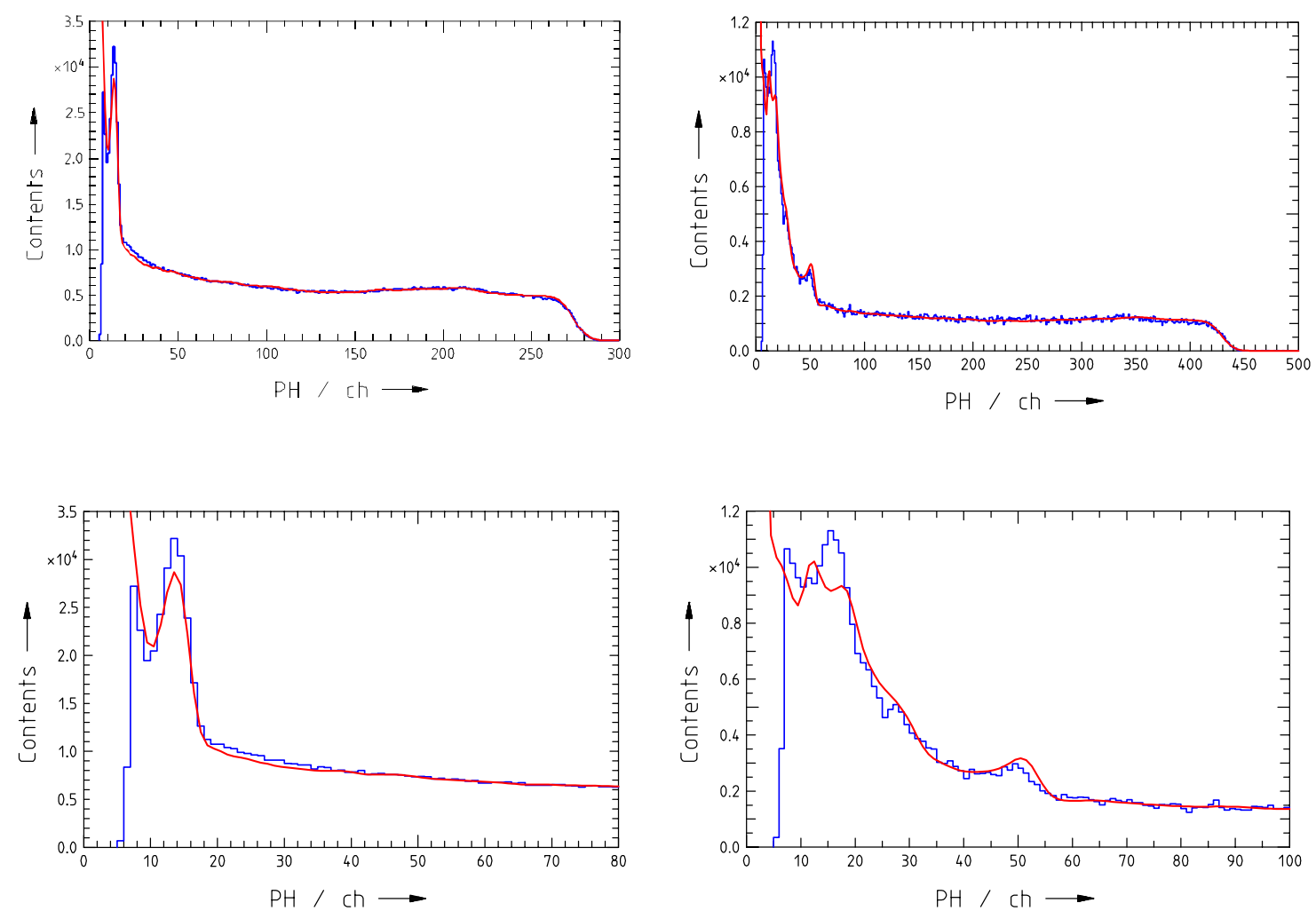

Figure 1: Comparison of response functions of an NE213-scintillator, $255 \mathrm{~mm}$ in diameter and $51 \mathrm{~mm}$ in length, for $9.9 \mathrm{MeV}$ (left) and $14.26 \mathrm{MeV}$ (right) neutrons, as measured (blue) and simulated (red) with version 7 of the NRESP-code [128]. Expanded plots of the low-amplitude regions are shown in the lower panels.

reproduced (see Fig. 1), the calculated efficiencies may exhibit deviations of $10 \%$ and more, depending on energy and threshold. The necessary corrections shown in Fig. 2 then result from the experimental responses fitted to the calculated response in the upper pulse height region.

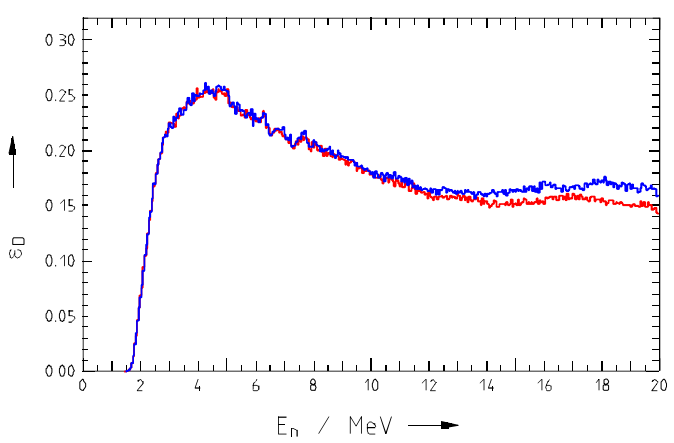

Figure 2: Neutron detection efficiency $\varepsilon_{\mathrm{D}}$ of an NE213 scintillator, $255 \mathrm{~mm}$ in diameter and $51 \mathrm{~mm}$ in length, for neutron energies up to $20 \mathrm{MeV}$ and a threshold of about $2 \mathrm{MeV}$

$\left(\mathrm{E}_{\text {eee }}>0.46 \mathrm{MeV}\right)$

(a) simulated (blue) with version 7 of the NEFFcode [128]

(b) simulation corrected for compliance with the experimental response in the low-amplitude region (red). 
Since this drawback of the MC simulations may not be solved in the near future, due to the lack of all necessary cross section data, the recommendation can only be, to establish the complete response matrix experimentally, e.g. by tof-spectrometry in neutron fields with a wide energy distribution and multiparameter data acquisition of tof-, pulse shape- and pulse height signals. The experimental response matrix, properly normalised with reference to the $n-p$ scattering cross section, may then be used, firstly, to calculate the neutron detection efficiency for any applied threshold, and secondly, in the deconvolution of pulse height spectra to obtain the spectral neutron fluences reliable in energy and absolute scale. In this way, neutron detection efficiencies may be determined which are comparable with high quality experimental calibrations such as those of Drosg [130].

\subsection{Time response and time resolution}

Organic scintillation detectors are well known for their excellent time resolution, especially when employed in coincidence experiments with gamma-sources, muons or high energy charged particles. Using the fastest plastic scintillators now available, a time resolution of less than 200 ps FWHM can be achieved for ${ }^{60} \mathrm{Co} \gamma-\gamma$-coincidences (see Fig. 26 in [71]), even when thresholds are set as low as $10 \%$ of the Compton edges. Time walk and jitter may increase slightly if the size of detector and/or light guide is increased, but the time resolution can still be better than $1 \mathrm{~ns}$ FWHM. In these experiments the time for depositing the energy and producing the light is short $(<<1 \mathrm{~ns})$ compared with the decay constants of the scintillator.

In case of neutron measurements, however, the time response is chiefly determined by the transit time of the neutrons within the detector (see Fig. 3 in [58]). Taking into account the detection mechanism and the timing procedure, calculated time response functions show some pecularities [131]. Assuming constant fraction timing, e.g. at $20 \%$ fraction, and taking into account, that the light produced by carbon recoil is heavily quenched and therefore insufficient to exceed the CFT-threshold, even though up to $23 \%$ of the neutron energy can be transferred to the recoil carbon, the time response is dominated by (see Fig. 3):

- the neutron flux attenuation, which shifts the effective centre towards the front of the detector and

- the multiple scattering of the neutrons on carbon, which extends the transit time before an interaction with hydrogen produces a signal exceeding the threshold. The tail may then extend up to $15 \mathrm{~ns}$ for large volume detectors and even overcompensate the effect of the flux attenuation such that the effective centre is shifted to the rear of the detector.

Folding the calculated time response functions with the normal distribution approximating the effect of time jitter (due to noise) and time walk (according to the dynamic range) a time response matrix can be established for the threshold actually used. This matrix may be applied for the analysis or the simulation of tof-experiments [131]. The effect of the detector geometry must be taken into account for tof-measurements at relatively small flight paths, e.g. for calibration measurements with ${ }^{252} \mathrm{Cf}$-sources [132,133]. 

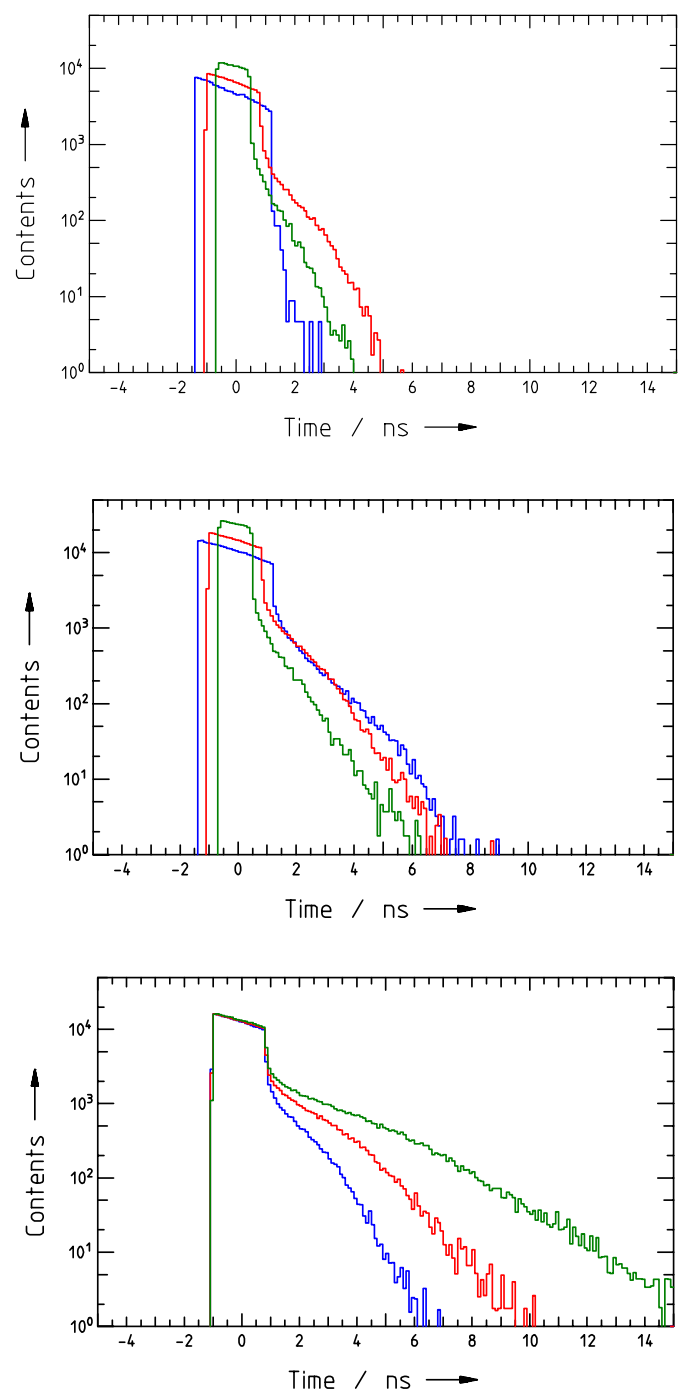

detector: NE213, 2" in length, 2" in diameter threshold: $\mathrm{E}_{\text {eee }}>0.5 \mathrm{MeV}$ neutron energy: $E_{n}=2 \mathrm{MeV}$ (blue), $4 \mathrm{MeV}$ (red) and $10 \mathrm{MeV}$ (green)

shift of mean value: $\mathrm{dt}=-0.21 \mathrm{~ns}$ (blue), $+0.00 \mathrm{~ns}$ (red) and $+0.02 \mathrm{~ns}$ (green)

detector: NE213, 2" in length, 2“ in diameter threshold: $\mathrm{E}_{\text {eee }}>0.2 \mathrm{MeV}$ neutron energy: $\mathrm{E}_{\mathrm{n}}=2 \mathrm{MeV}$ (blue), $4 \mathrm{MeV}$ (red) and $10 \mathrm{MeV}$ (green) shift of mean value: $\mathrm{dt}=-0.03 \mathrm{~ns}$ (blue), $+0.06 \mathrm{~ns}$ (red) and +0.03 ns (green)

detector: NE213, 2" in length, and 2“ (blue), 4" (red), 10" (green) in diameter threshold: $\mathrm{E}_{\text {eee }}>0.2 \mathrm{MeV}$ neutron energy: $\mathrm{E}_{\mathrm{n}}=4 \mathrm{MeV}$ shift of mean value: $\mathrm{dt}=+0.04 \mathrm{~ns}$ (blue), $+0.12 \mathrm{~ns}$ (red), +0.46 ns (green)

Figure 3: Simulation of the time response with version 7 of the NRESP-code [128] for NE213 scintillation detectors and a constant fraction timing at $20 \%$ fraction as a function of detector size, neutron energy and electron threshold. The centre of the scintillator along the cylinder axis marks the time zero reference. The means of these time distributions correlate with the effective centre defining the flight path to the neutron point source.

(Note: $1 "=25.4 \mathrm{~mm}$ )

\subsection{Pulse shape discrimination capability}

The capability of some organic scintillators to distinguish between gamma- and neutroninduced events by means of different pulse shapes is well-known and has been utilized since the late nineteen-fifties [134,135]. The pulse shape discrimination (PSD) technique is based on the fact that the light output of the well-known organic scintillators consists of two main components: a fast, exponentially decaying component, of lifetime typically in the range 2-30 
ns, and a relatively slow non-exponential component that may extend the "tail" of the scintillation to $300-600 \mathrm{~ns}[110,136]$. For certain crystal and liquid scintillators the fraction of light emission in the slow component can be $10-30 \%$ for strongly ionizing particles. For these scintillators and for charged particles that are brought to rest in the scintillator, the proportion of light emission in the tail component depends on the type (mass and charge) and energy of the particle producing the scintillation. This dependence provides the basis for particle identification by means of PSD. Many different methods and techniques have been proposed and used to implement PSD and the PSD method is now widely used for neutron-gamma discrimination, that is discrimination between Compton electrons released by gamma rays and recoil protons or heavier charged particles released by neutron interactions in organic scintillators [137,138]. For most plastic scintillators the proportion of light in the slow component is typically $<3 \%$, hence the PSD effect is much smaller and usually insufficient to be of practical value. In the case of liquid scintillators the triplet excitations that are responsible for the slow component [110] are quenched if dissolved oxygen is present in the liquid. To obtain good PSD this oxygen must be removed or displaced, for example by bubbling dry nitrogen or argon through the scintillator and then sealing it off from the atmosphere.

Reviews of early work on PSD and PSD systems are available in Refs. [4,139]. Recent and early work of special interest and importance is presented in Refs. [88,140-147]. The $n / \gamma-$ discrimination capability can be quantified by a figure-of-merit $M$ which is defined as the ratio of the separation $S$ of the $\gamma$ - and n-branches divided by the sum of their widths $\Gamma_{\gamma}$ and $\Gamma_{\mathrm{n}}$. and deduced from two-parameter data of pulse shape vs total pulse height (see for example Fig. 4 of [144]). The figure-of-merit can then be determined as a function of the total light output $L$ as $M(L)=S(L) /\left(\Gamma_{\gamma}(L)+\Gamma_{\mathrm{n}}(L)\right)$. In this way, the properties of different PSD systems may be compared and the influence of adjustable parameters of the PSD system investigated. For example, the reduction of the integration time from $500 \mathrm{~ns}$ to $100 \mathrm{~ns}$ considerably reduces the figure-of-merit for a certain light output, as shown in Fig. 5 of [144].

A topical field at present is the development of digital PSD systems based on the use of flash ADCs and commercially available waveform analysers [16,25,140,148-151]. It is expected that these systems will be capable of processing count rates up to $\mathrm{MHz}$ for fast counting and for spectrometry, without considerable decrease of the energy resolution. They also offer increased flexibility, for example by making it possible to implement PSD, pileup-rejection and other features through the use of software [120,152].

\subsection{Energy resolution in time-of-flight spectrometry and unfolding methods}

Organic scintillation detectors are frequently used for neutron spectrometry, in particular those detectors with $\mathrm{n} / \gamma$-discrimination capability are employed for applications in mixed fields. The achievable energy resolution depends to a large extent on the quality of the characterisation of the detector system.

In time-of flight spectrometry, the properties of the pulsed or tagged neutron source, e.g. a pulsed beam or a fission detector, may sometimes be deduced from the well separated peak originating from prompt gamma-rays produced in the source. This intrinsic resolution function 
including the source properties as well as time jitter and time walk of the detector system (provided the pulse height distributions of gamma- and neutron-induced events are comparable) can then be used to fold the calculated time response for neutrons in order to establish a time response matrix for the actual threshold applied, which in turn may be used for unfolding measured tof-spectra. In principle, a significantly improved energy resolution can be achieved, if measured tof-spectra are properly deconvoluted. Inclusion of the time response matrix in the simulation of the tof-spectra for comparison with measured spectra of scattered neutrons was successfully applied to separate overlapping peaks [131].

Similarly, the unfolding of gamma- and neutron-induced pulse height spectra, well separated by pulse shape analysis if taken in mixed fields, can result in an energy resolution that is a factor of 4-5 times better (smaller) than the pulse height resolution of the corresponding Compton- or recoil proton-edges which define the gamma or neutron energy [60]. An indispensable precondition for obtaining neutron spectra well-defined in energy and width is, however, that the response matrix has been properly determined, either experimentally with correct normalisation or by $\mathrm{MC}$ simulation folded with an adequate pulse height resolution function. Using modern unfolding procedures [153-155] the energy resolution is chiefly limited by the statistics of the measured spectra, as demonstrated for pure photon spectra [60], or by the statistics of the measured response matrix [120]. For more details, we refer to recent publications, e.g. in the special issues NIM A476 (2002) [156] and RPD 107 (2003) [157] with a comprehensive description of neutron spectrometry including a detailed discussion of unfolding algorithms.

In conclusion it can be stated, that the energy resolution chiefly depends on the quality of the corresponding response matrices applied for the analysis of tof or pulse height spectra.

\section{Present status and future trends}

\subsection{Present status and applications}

Below $200 \mathrm{keV}$, Li-glass scintillators, loaded with up to $7.7 \%{ }^{6} \mathrm{Li}$ in weight, are often used for time-of-flight spectrometry. Although the ${ }^{6} \mathrm{Li}(\mathrm{n}, \alpha)$-cross section exhibits a smooth $1 / v$ behaviour up to the resonance at $250 \mathrm{keV}$, the efficiency must carefully be calculated to account for the resonant structure of the neutron interaction with the major constituents of the detector in this energy region. The strongly energy-dependent corrections reflect the influence of silicon and oxygen, even if thin scintillators are employed in a low-mass environment $[34,38]$. Nevertheless, these detectors have been successfully employed in tof-measurements for energies up to $1(2) \mathrm{MeV}$ [38]. The sensitivity of ${ }^{6} \mathrm{Li}$-glass scintillators to gamma rays can sometimes be a problem, but this can be overcome by fitting the shape of the underlying background or operating the ${ }^{6} \mathrm{Li}$-glass in conjuction with an identical ${ }^{7} \mathrm{Li}$-glass, which is then used to monitor and subtract the gamma component [35,37].

For energies of about $20 \mathrm{keV}$ to $5 \mathrm{MeV}$, any kind of organic scintillator can be used. Renner et al. [158] have demonstrated that pulse height spectra taken for mono-energetic 
neutrons in this range, using plastic scintillators of various thickness, are in excellent agreement with MC-simulations made using the O5S-code [159], both in shape and absolute scale. Provided low-mass encapsulation is used these detector systems can be regarded as metrological reference systems because the cross sections needed for the response calculations are among the best known reference data available and have an almost smooth energy dependence in this energy region except for a few resonances in the scattering cross section for carbon. Spectral fluence measurements with uncertainties less than $2 \%$ are possible if the photon background can be strongly suppressed, e.g. in tof-measurements.

For the same reason, the neutron response can be reliably calculated for a "black" detector as designed by W.P. Pönitz [160]. Detection losses due to backscattering at the recessed entrance aperture of this detector are almost negligible if a well-collimated neutron beam is used. Since the neutron detection efficiency exceeds $90 \%$ for neutron energies between 0.5 $\mathrm{MeV}$ (a typical threshold that permits good pulse shape discrimination against gamma background) and $8 \mathrm{MeV}$, the efficiency can be calculated with uncertainties $<1 \%$. However, even for mono-energetic neutrons broad pulse height spectra result from multiple neutron scattering in the voluminous detector, chiefly caused by the non-linear light output response of the liquid scintillator. Nevertheless, background can be well discriminated against the neutroninduced events by means of a simple threshold. The calculated response of a similarly designed "black" detector of NIST (formerly NBS) were confirmed in absolute scale for $2.3 \mathrm{MeV}$ neutrons by applying the time-correlated associated particle (tcap) method for calibration [161]. Both of these black detector systems were successfully applied to relate the cross section for neutron-induced fission of ${ }^{235} \mathrm{U}$ to the total $n$-p-scattering cross reaction, which is regarded as the reference standard. For higher neutron energies the efficiency decreases because the n-p cross section decreases and reactions on carbon, which do not result in a detectable light output, cause a loss of neutron flux. These effects increase the uncertainty in the prediction of the detection efficiency.

Another method for determining neutron fluence in the energy range up to $15 \mathrm{MeV}$ and by reference to the total n-p cross section is based [162] on the use of coincidences between a pair of thin plastic scintillators, each $2.57 \mathrm{~mm}$ thick and stacked close together, optically separated by a thin aluminium foil. This geometry suppresses the response due to multiple scattering and ensures that, even for $14 \mathrm{MeV}$ neutrons, the summed pulse height spectrum for coincident events chiefly reflects the angular distribution of neutrons scattered only once on a hydrogen nucleus in the upstream scintillator. The calculated efficiency was also confirmed to better than $1 \%$ by absolute calibration measurements using the tcap-method for the reactions $\mathrm{D}(\mathrm{d}, \mathrm{n})^{3} \mathrm{He}$ or $\mathrm{T}(\mathrm{d}, \mathrm{n})^{4} \mathrm{He}$ for neutrons with energies of $2.5 \mathrm{MeV}$ or $14 \mathrm{MeV}$ respectively.

Essentially the same principle was applied for neutron spectrometry, both by means of tofmeasurements as well as by unfolding of pulse height spectra, in the energy range from $50 \mathrm{MeV}$ to $150 \mathrm{MeV}$ [90,91]. Correlated signals of two or three liquid scintillators, each $7 \mathrm{~cm}$ in thickness and closely stacked, were analysed using pulse shape discrimination to reject gammaevents. The response matrix was determined experimentally and normalised to $n-p$ backscattering at the upper edge of the recoil proton distributions. Spectral neutron fluences 
were determined by means of tof-spectrometry and, independently, by unfolding the simultaneously measured pulse height spectra. Excellent agreement was achieved [91] between the results obtained by the two different methods, thus demonstrating that pulse-height unfolding is a viable method for determining spectral neutron fluence up to $150 \mathrm{MeV}$.

Another method that uses response functions for full energy deposition by neutrons within the detector is the double-pulse [20] or capture-gated neutron spectrometer. This spectrometer is based on the combination of neutron moderation in an organic scintillator and capture of the thermalised neutrons in either the same scintillator or an associated separate scintillator $[14,28,163,164]$. The scintillator in which neutron capture takes place incorporates a small concentration of a nuclide such as ${ }^{6} \mathrm{Li},{ }^{10} \mathrm{~B}$ and/or ${ }^{155,157} \mathrm{Gd}$, which has a large capture cross section for thermal neutrons. The integrated signal of the fully moderated neutrons can be separated from all other events, in particular the gamma-background, by tagging with the considerably delayed capture signal which can either be identified by the time correlation and/or the different pulse shape. Similar to black detectors, the response can be reliably calculated, but also for medium neutron energies only [164].

A disadvantage of organic scintillators when proton recoils produced by high energy $(>100 \mathrm{MeV})$ neutrons are being detected is that large volumes are needed to limit the escape of recoil protons from the scintillator (wall effect) due to their long range; for example, the range of a $100 \mathrm{MeV}$ proton in liquid scintillator is about $9 \mathrm{~cm}$, therefore a scintillator with linear dimensions $>20 \mathrm{~cm}$ should be used for spectrometry of $100 \mathrm{MeV}$ neutrons. For dimensions less than the maximal range, the response functions for recoil protons are no more unique and pulse shape discrimination capability is lost for the highest energy proton recoils. Various methods have been proposed to identify and discard signals suffering wall effects. For example the scintillator may be encapsulated by a thin inorganic scintillator with much higher density [165]. Taking advantage of the different pulse shapes of inner liquid scintillator and outer crystal, any signals with contributions from the outer detector can be identified in this phoswicharrangement by pulse shape analysis. Since signals from external high-energy charged particles can also be vetoed, versatile applications are possible, e.g. for neutron spectrometry in aircraft, satellites or space missions.

The drawback of increasing wall-effects in organic scintillators with increasing neutron energy can also be avoided by employing scintillators with higher density, i.e. inorganic scintillators. CsI(Tl)-scintillators are often used as $E$-detector in recoil proton telescopes. Very recently $[95,166]$, the response of a $17 \mathrm{~cm}$ thick $\mathrm{BaF}_{2}$-crystal was calibrated for neutron energies up to $200 \mathrm{MeV}$. While the responses calculated with the FLUKA-code considerably underestimated the experimental data, the experimental efficiencies were interpolated for the normalisation of tof-spectra. This detector system was then successfully applied for the determination of the spectral neutron fluence associated with heavy ion reactions [167].

Sets of long plastic or liquid scintillators are often used for tof-spectrometry in rather complex arrangements. Even for $3 \mathrm{~m}$ long scintillators, excellent time resolution $(<1 \mathrm{~ns})$ is achieved for muons or high-energy charged particles if the time difference of two signals from PMTs at both ends is used for correction or identification of the position of interaction. The 
correlated tof-spectrometry of neutrons and protons from the dissociation of deuterons by high energy photons is an exceptional example for an application of such large detector systems [94].

The great variety of applications of scintillation detectors in neutron measurements prevents a complete review. Very special systems were designed applying gaseous [168] and liquid [77] ${ }^{3} \mathrm{He}$-counters for tof-spectrometry below and above $1 \mathrm{MeV}$ respectively. Scintillating fibres are increasingly employed, e.g. as position-sensitive neutron converters in optical readout-systems for fast neutron radiography [169-171]. Liquid scintillation detectors were successfully employed in the neutron diagnostics of plasma discharges, both for fast counting in horizontal and vertical profile cameras [172] and for high-resolution spectrometry as well $[63,65,120]$.

\subsection{Future trends}

Organic scintillation detectors with pulse shape discrimination capabilities are employed when background due to gamma-rays has to be eliminated. Except for low $(<200 \mathrm{keV})$ and high $(>200 \mathrm{MeV})$ energies this type of scintillation detector will also in future be the first choice when neutron detectors are designed for new applications. Major efforts are presently being made to overcome some of the well known drawbacks of organic scintillation detector systems, namely:

- the limitation in processing high count rates chiefly due to the deadtime of conventional nuclear electronics needed for spectrometry and pulse shape analysis. This problem will be solved when appropriate waveform analysers are available. Ideally, the fast detector signals should be sampled at $>500 \mathrm{MHz}$ with $>10$ bit resolution and stored event by event for offline analysis or, even better, fast online interpretation. Besides faster processors and larger memories, appropriate algorithms have to be developed for particular applications,

- the gain instabilities of photomultiplier caused by varying magnetic fields, variable count rates and temperature. Besides efficient corrections by feeding in stabilised LED or laser signals [173-176], the aim is to use other photon-sensitive detectors with low noise instead of PMTs, e.g. Si-diodes [177] which should be insensitive to harsh environmental conditions.

- the hazard of liquid scintillators due to their basic material, e.g. xylene. The recently introduced gelled scintillator [44] should be an ideal solution, if the material exhibits reasonable pulse height resolution and $\mathrm{n} / \gamma$-discrimination properties. First results are very encouraging [45].

- the long range of high-energy recoil protons in organic material due to their low density and light constituents which cause considerable wall effects. Although different techniques can be employed to discriminate such events (phoswich or tagged scintillators), inorganic scintillators with much higher density should be selected for optimal neutron detection efficiency taking into consideration the relevant reactions leading to light emission. These detectors must then be carefully characterised both experimentally and by means of advanced simulation codes. Ideally, the detector should have the capability to separate neutron- and gamma-induced events either by their amplitude or by means of pulse shape 
differences. Considerable progress has been achieved in developing new inorganic scintillators $[12,13,100,178]$, in particular increasing the light output and decreasing the decay times by optimised doping. Perhaps $\mathrm{PbWO}_{4}$-crystals [103] will be found to be well suited for fast neutron detection.

\section{Acknowledgement}

The authors gratefully acknowledge the invitation by the scientific committee for participation in the workshop and presentation of this contribution and the support of Dr. Dankwart Schmidt at PTB who provided us with unpublished experimental data and the corresponding Monte Carlo simulations.

\section{References}

[1] J.A. Harvey, N.W. Hill, Scintillation detectors for fast neutron physics, Nucl. Instr. Meth. 162 (1979) 507-530

[2] D.A. Bromley Ed., Detectors in Nuclear Science, Nucl. Instr. Meth. 162 (1979)

[3] F.W.K. Firk, Neutron time-of-flight spectrometers, Nucl. Instr. Meth. 162 (1979) 539-563

[4] F.D. Brooks, Development of organic scintillators, Nucl. Instr. Meth. 162 (1979) 477-506

[5] J.B. Marion, J.L. Fowler Eds., Fast Neutron Physics; Part 1: Techniques; Part 2: Experiments and Theory, Interscience, New York, 1960

[6] G.F. Knoll, Radiation Detection and Measurement, John Wiley \& Sons, New York, 2000, 3rd Ed.

[7] J.B.A. England, Techniques in Nuclear Structure Physics, MacMillan, London, 1974.

[8] J.B. Birks, The theory and practice of scintillation counting, Pergamon Press, London, 1964

[9] F.D. Brooks, Neutron detectors and spectrometers, SPIE 2867 (1997) 538-549

[10] A.J. Peurrung, Recent developments in neutron detection, Nucl. Instr. Meth. A443 (2000) 400415

[11] F.D. Brooks, H. Klein, Neutron spectrometry - historical review and present status, Nucl. Instr. Meth. A476 (2002) 1-11

[12] S.E. Derenzo, M-J. Weber, E. Bourret-Courchesne, M.K. Klintenberg, The quest for ideal inorganic scintillators, Nucl. Instr. Meth. A505 (2003) 111-117

[13] R. Novotny, Inorganic scintillators - a basic material for instrumentation in physics, Nucl. Instr. Meth. A537 (2005) 1-5

[14] G.I. Britvich et al, A neutron detector on the basis of a boron-containing plastic scintillator, Nucl. Instr. Meth. A550 (2005) 343-358

[15] S. Ait-Boubker, M. Avenir, G. Bagieu et al, Thermal neutron detection and identification in a large volume with a new lithium-6 loaded liquid scintillator, Nucl. Instr. Meth. A277 (1989) 461-466

[16] R. Aleksan, J. Bouchez, M. Boussicut et al, Pulse shape discrimination with a $100 \mathrm{MHz}$ flash ADC system, Nucl. Instr. Meth. A273 (1988) 303-309

[17] R. Aleksan, J. Bouchez, M. Cribier et al, Measurement of fast neutrons in the Gran Sasso laboratory, Nucl. Instr. Meth. A274 (1989) 203-206

[18] M. Abbes, B. Achkar, S. Ait-Boubker et al, The Bugey 3 neutrino detector, Nucl. Instr. Meth. A374 (1996) 164-187

[19] E.R. Rae, E.M. Bowey, A scintillation detector for neutrons of intermediate energy, Proc. Phys. Soc. A66 (1953) 1073-1075

[20] C.O. Muehlhause, Neutron detection by reactions induced in scintillators, in Fast Neutron Physics Part 1 (ed J.B. Marion \& J.F. Fowler), Interscience, New York, 1960, p 387-411 
[21] L.M. Bollinger, G.E. Thomas, Boron-loaded liquid scintillation neutron detectors, Rev. Sci. Instr. 28 (1957) 489-496

[22] H.E. Jackson, G.E. Thomas, Boron-loaded neutron detector with very low $\gamma$-ray sensitivity, Rev. Sci. Instr. 36 (1965) 419-425

[23] S.C. Wang, C.C. Hsu, R.W.S. Leung et al, A feasibility study of boron-loaded liquid scintillator for the detection of electron anti-neutrinos, Nucl. Instr. Meth. A432 (1999) 111 121

[24] A.H.D Rasolonjatovo, T. Shiomi, E. Kim, T. Nakamura, T. Nunomiya, A. Endo, Y. Yamaguchi, M. Yoshizawa, Development of a new neutron monitor using a boron-loaded organic liquid scintillation detector, Nucl. Instr. Meth. A492 (2002) 423-433

[25] S.D. Jastaniah, P.J. Sellin, Techniques for $n / \gamma$ pulse shape discrimination and capture-gated neutron spectroscopy using liquid scintillators, Nucl. Instr. Meth. A517 (2004) 202-210

[26] T. Aoyama, K. Honda, C. Mori, N.Takeda, Energy response of a full-energy-absorption neutron spectrometer using boron-loaded liquid scintillator BC-523, Nucl. Instr. Meth. A333 (1993) 492-501

[27] H.P. Chou, C.Y. Horng, Integral test of a boron-10 loaded liquid scintillator for neutron detection, Nucl. Instr. Meth. A328 (1993) 522-525

[28] E.A. Kamykowski,Comparison of calculated and measured spectral response and intrinsic efficiency for a boron-loaded plastic scintillator, Nucl. Instr. Meth. A317 (1992) 559-566

[29] A. Gavron, The role of science in treaty verification, Appl. Rad. Isotop. 63 (2005) 607-611

[30] S. Normand, B. Mouanda, S. Haan, M. Louvel, Discrimination methods between neutrons and gamma rays for boron-loaded plastic scintillators, Nucl. Instr. Meth. A484 (2002) 342-350

[31] M.C. Miller, R.S. Biddle, S.C. Bourret et al., Neutron detection and applications using a BC454/BGO array, Nucl. Instr. Meth. A422 (1999) 89-94

[32] R.B. Murray, Use of ${ }^{6} \mathrm{LiI}(\mathrm{Eu})$ as a scintillation detector and spectrometer for fast neutrons, Nucl. Instr.Meth. 2 (1958) 237-248

[33] F.W.K. Firk, G.C. Slaughter, R.J. Ginther, An improved ${ }^{6}$ Li-loaded glass scintillator for neutron detection, Nucl. Instr. Meth. 13 (1961) 313-318

[34] R.L. Macklin, N.W. Hill, B.J. Allen, Thin ${ }^{6} \operatorname{Li}(n, \alpha)$ T transmission flux monitor, Nucl. Instr. Meth. 96 (1971) 509-513

[35] R. Aryaimedad et al., Palm-size low-level neutron sensor for radiation monitoring, IEEE NS43 (1996) 1539-1543

[36] G.B. Spector, T. McCollum, A.R. Spowart, Scintillator fiber optic long counter response to neutrons from 0.5-15.1MeV, Nucl. Instr. Meth. A346 (1994) 273-278

[37] M. Asghar, F.D. Brooks, Neutron resonance scattering measurements with ${ }^{6}$ Li-glass detectors, Nucl. Instr. Meth. 39 (1966) 68-76

[38] D.B. Gayther, A measurement of the ${ }^{6} \mathrm{Li}(n, a)$-cross section, Annals of Nuclear Energy 4 (1977) 515-526

[39] P. Ottonello, V. Palestrini, G.A. Rottigni, G. Zanella, R. Zannoni, Slow neutron beam diagnostics with a scintillating fiber detector, Nucl. Instr. Meth. A366 (1995) 248-253

[40] K.H. Abel, R.J. Arthur, M. Bliss et al., Scintillating-glass-fiber neutron sensors, Nucl. Instr. Meth. A353 (1994) 114-117

[41] F.D. Smit, F.D. Brooks, Angular distribution of neutrons from $D(\gamma, n) H$ at $E_{\gamma}=2.75 \mathrm{MeV}$, Nucl. Phys. A465 (1987) 429-444

[42] A. Takahashi, I. Murata, Kokoo, S. Ogino, Y. Murakami, H. Nishizawa, T. Kondo, A time-offlight spectrometer with pulse shape discrimination for the measurement of double-differential charged-particle emission crossections, Nucl. Instr. Meth. A401 (1997) 93-103

[43] D.A. Roberts, F.D. Bechetti, K. Ashktorab et al., Deuterated liquid scintillator (NE230) as a fast neutron detector for cold-fusion and other research, IEEE Transactions on Nuclear Science 39 (1992) 532-535

[44] G. Jonkmans et al., A Canadian high-energy neutron spectrometry system for measurements in space, Acta Astronautica 56 (2005) 975-979

[45] M.B. Smith, H.R. Andrews, L.G.I. Bennett et al, Canadian high-energy neutron spectrometry system (CHENSS), This Workshop (2006) paper OA11 
[46] M. Moszynski, Light pulse shape study for crystal organic scintillators, Nucl. Instr. Meth. 153 (1978) 439-443

[47] F.D. Brooks, W.A. Cilliers, B.R.S. Simpson, F.D. Smit, M.S. Allie, D.T.L. Jones, W.R. McMurray, J.V. Pilcher, Deuterated anthracene spectrometer for 5-30MeV neutrons, Nucl. Instr. Meth. A270 (1988) 149-156

[48] W. Hansen, D. Richter, Determination of light output function and angle dependent correction for a stilbene crystal scintillation neutron spectrometer, Nucl. Instr. Meth. A476 (2002) 195199

[49] F. Cvachovec, J. Cvachovec, P. Talovsky, Anisotropy of light output in response of stilbene detectors, Nucl. Instr. Meth. A476 (2002) 200-202

[50] W. Tornow, W. Arnold, J. Herdtweck, G. Mertens, Measurement of the response of the deuterated scintillators NE232 and NE230 to protons and deuterons, Nucl. Instr. Meth. A244 (1986) 477-482

[51] S. Croft, J.M.Adams, D.S. Bond, N.P. Hawkes, N. Watkins, A measurement of the light output function of the deuterated liquid scintillator NE230 to recoil deuterons with energies between 0.62 and 14.5 MeV, Nucl. Instr. Meth. A316 (1992) 324-332

[52] A.A. Naqvi, F.Z. Khiari, A. Aksoy, A. Coban, A.M. Al-Jalal, Light yield measurements of an NE213 detector for 2.8-14.8 MeV neutrons, Nucl. Instr. Meth. A325 (1993) 574-577

[53] D. Schmidt, A. Asselineau, R. Böttger, H. Klein, L. Lebreton, S. Neumann, R. Nolte, G. Pichenot, Characterisation of liquid scintillation detectors, Nucl. Instr. Meth. A476 (2002) 186-189

[54] N.P. Hawkes, J.M. Adams, D.S. Bond, S. Croft, O.N. Jarvis, N. Watkins, Measurements of the proton light output function of the organic liquid scintillator NE213 in several detectors, Nucl. Instr. Meth. A476 (2002) 190-194

[55] A. Hildebrand, H. Park, S. Khurana, R. Nolte, D. Schmidt, Experimental determination of the response matrix of a BC501 scintillation detector using a wide neutron spectrum: a status report, PTB-6.42-05-1, Braunschweig, 2005

[56] C. Weber, I. Fabry, V. Huhn, A. Siepe, W. von Witsch, Accurate determination of the fastneutron detection efficiency for organic scintillators using a collimated neutron beam, Nucl. Instr. Meth. A488 (2002) 307-313

[57] F. Arneodo et al., Calibration of BC501A liquid scintillator cells with monochromatic neutron beams, Nucl. Instr. Meth. A418 (1998) 285-299

[58] J.R.M. Annand, R.W. Finlay, M.A. Polster, Timing characteristic of large cylindrical NE213 counters, Nucl. Instr. Meth. A234 (1985) 483-487

[59] F.E. Cecil, J.C. Scorby, Investigation of the neutron detection properties of the fast liquid scintillator BC505, Rev. Sci. Instrum. 63 (1992) 4559-4561

[60] H. Klein, S. Neumann, Neutron and photon spectrometry with liquid scintillation detectors in mixed fields, Nucl. Instr. Meth. A476 (2002) 132-142

[61] N.A. Lurie, L. Harris, J.C. Young, Calculation of gamma-ray response matrix for $5 \mathrm{~cm}$ NE213 organic liquid scintillation detector, Nucl. Instr. Meth. 129 (1975) 543-555

[62] J.W. Bennett, M.C. Scott, Fast neutron spectrum measurements in anthropomorphic phantoms using an NE213 scintillation spectrometer, Phys. Med. Biol. 32 (1987) 1645-1648

[63] J.K. Dickens, N.W. Hill, F.S. Hou, J.W. McConnell, R.R. Spencer, T.Y. Tsang, An NE213 scintillator-based neutron detection system for diagnostic measurements of energy spectra for neutrons having energies $>0.8 \mathrm{MeV}$ created during plasma operations at the Princeton Tokamak Fusion Test Reactor, ORNL/TM-9561, Oak ridge, 1985

[64] Yu.A. Kaschuck, B. Esposito, L.A. Trykov, V.P. Semenov, Fast neutron spectrometry with organic scintillators applied to magnetic fusion experiments, Nucl. Instr. Meth. A476 (2002) 511-515

[65] B. Esposito et al., Neutron measurements on JET using an NE213 scintillator with digital pulse shape discrimination, Rev. Sci. Instr. 75 (2004) 3550-3552

[66] F.L. Lynch, Basic limitation of scintillation counters in time measurements, IEEE NS-22 (1975) 58-64 
[67] S. Mouatassim, G.J. Costa, G. Guillame, B. Heusch, A. Huck, M. Moszynski, The light yield response of NE213 organic scintillators to charged particles resulting from neutron interactions, Nucl. Instr. Meth. A359 (1995) 530-536

[68] B. Bengtson, M. Moszynski, Timing properties of scintillation counters, Nucl. Instr. Meth. 81 (1970) 109-120

[69] M. Moszynski, B. Bengtson, Light pulse shapes from plastic scintillators, Nucl. Instr. Meth. 142 (1977) 417-434

[70] B. Bengtson, M. Moszynski, Study of primary energy transfer process in ultrafast plastic scintillators, Nucl. Instr. Meth. 155 (1978) 221-231

[71] M. Moszynski, B. Bengtson, Status of timing with plastic scintillation detectors, Nucl. Instr. Meth. 158 (1979) 1-31

[72] B. Bengtson, M. Moszynski, Timing improved by the use of dynode signals studied with different scintillators and photomultipliers, Nucl. Instr. Meth. 204 (1982) 129-140

[73] C. Wu et al., The timing properties of a plastic time-of-flight scintillator from a beam test, Nucl. Instr. Meth. A555 (2005) 142-147

[74] P. Doll, G. Fink, F.P. Brady, R. Garrett, H.O. Klages, H. Krupp, dE-E telescope systems for detecting charged particles produced with a "white"neutron beam, Nucl. Instr. Meth. A250 (1986) 526-533

[75] R.C. Byrd, P.L. McGaughey, W.C. Sailor, R.C. Hammock, Y. Yariv, Measurements and calculations of the performance of a multi-element neutron detector, Nucl. Instr. Meth. A313 (1992) 437-451

[76] M.W. McNaughton, F.P. Brady, W.B. Broste, A.L. Sagle, S.W. Johnsen, An investigation of the neutron detection efficiency of a plastic scintillation detector in the energy range 6 to 41 $\mathrm{MeV}$, Nucl. Instr. Meth. 116 (1974) 25-28

[77] R. van Staa, J. Reher, B. Zeitnitz, A liquid He-3 detector for fast neutrons, Nucl. Instr. Meth. 136 (1976) 241-248

[78] H.O. Klages, W. Heeringa, H. Dobiasch, R. Fischer, B. Haesner, P. Schwarz, J. Wilczynski, B. Zeitnitz, Analyzing power of the n-He3 elastic scattering from 3.7 to $22.0 \mathrm{MeV}$, Nucl. Phys. A443 (1985) 237-248

[79] R. Nath, F.W.K. Firk, R.J. Holt, H.L. Schultz, Polarization studies using the spin-precession method with a continuous energy spectrum of neutrons, Nucl. Instr. Meth. 98 (1972) 385-391

[80] E. Aprile, P. Cushman, K. Ni, P. Shagin, Detection of liquid xenon scintillation light with a silicon photomultiplier, Nucl. Instr. Meth. A556 (2005) 215-218

[81] C.M. Bartle, R.C. Haight, Small inorganic scintillators as neutron detectors, Nucl. Instr. Meth. A422 (1999) 54-58

[82] T. Matulewicz et al.,Response of $\mathrm{BaF}_{2}, \mathrm{CsI}(\mathrm{Tl})$ and Pb-glass detectors to neutrons below 22 MeV, Nucl. Instr. Meth. A274 (1989) 501-506

[83] R. Nolte, H.J. Brede, U.J. Schrewe, H. Schuhmacher, Neutron spectrometry with liquid scintillation detectors at neutron energies between $20 \mathrm{MeV}$ and $70 \mathrm{MeV}$ : a status report, PTBN-9, Braunschweig, 1993

[84] N. Nakao et al., Measurements of response function of organic liquid scintillator for neutron energy range up to $135 \mathrm{MeV}$, Nucl. Instr. Meth. A362 (1995) 454-465

[85] S. Meigo, Measurements of the response function and the detection efficiency of an NE213 scintillator for neutrons between 20 and 65MeV, Nucl. Instr. Meth. A401 (1997) 365-378

[86] N. Nakao, T. Kurosawa, T. Nakamura, Y. Uwamino, Development of a quasi-monoenergetic neutron field and measurements of the response function of an organic liquid scintillator for the neutron energy range from 66 to $206 \mathrm{MeV}$, Nucl. Instr. Meth. A476 (2002) 176-180

[87] J. Thun, J. Blomgren et al., The response of a liquid scintillator detector to $21-100 \mathrm{MeV}$ neutrons, Nucl. Instr. Meth. A478 (2002) 559-576

[88] R.St. Onge, A. Galonsky, R.K. Jolly, T.M. Amos, Organic-scintillator pulse-shape discriminator signatures associated with high-energy neutrons, Nucl. Instr. Meth. 126 (1975) 391-395

[89] D.T.L. Jones, J.E. Symons, T.J. Fulcher, F.D. Brooks, M.R. Nchodu, M.S. Allie, A. Buffler, M.J. Oliver, Neutron fluence and kerma spectra of a $p(66) / B e(40)$ clinical source, Med. Phys. 19 (1992) 1285-1291 
[90] A. Buffler, F.D. Brooks, M.S. Allie, P.J. Binns, V. Dangendorf, K.M. Langen, R. Nolte, H. Schuhmacher, Measurement of neutron energy spectra from 15 to $150 \mathrm{MeV}$ using stacked liquid scintillators, Nucl. Instr. Meth. A476 (2002) 181-185

[91] F.D. Brooks, M.S. Allie, A. Buffler, V. Dangendorf, M.S. Herbert, S.A. Makupula, R. Nolte, F.D. Smit, Measurement of neutron fluence spectra up to $150 \mathrm{MeV}$ using a stacked scintillator neutron spectrometer, Rad. Protect. Dosim. 110 (2004) 151-155

[92] W.B. Amian, M.M.Meier, R.C. Byrd, C.A. Goulding, G.L. Morgan, C.E. Moss, Efficiency calibration of a cylindrical BC418 neutron detector at neutron energies between 135 and 800 $\mathrm{MeV}$, Nucl. Instr. Meth. A313 (1992) 452-456

[93] C.D. Goodman, J. Rapaport, D.E. Bainum, C.E. Brient, Large, time-compensated scintillation counters for high-energy neutron tof-measurements, Nucl. Instr. Meth. 151 (1978) 125-134

[94] P. Grabmayr, T. Hehl, A. Stahl, J.R.M. Annand, R.O. Owens, A high-resolution, large acceptance scintillation time-of-flight spectrometer, Nucl. Instr. Meth. A402 (1998) 85-94

[95] K. Gunzert-Marx, D. Schardt, R.S. Simon, F. Gutermuth, T. Radon, V. Dangendorf, R. Nolte, Response of a BaF2 scintillation detector to quasi-monoenergetic fast neutrons in the range of 45 to $198 \mathrm{MeV}$, Nucl. Instr. Meth. A536 (2005) 146-153

[96] R.A. Kryger, A. Azhari, E. Ramakrishnan, M. Thoennessen, S. Yokoyama, Efficiency of a $\mathrm{BaF}_{2}$ scintillator detector for 15-150 MeV neutrons, Nucl. Instr. Meth. A346 (1994) 544-547

[97] V. Wagner et al., Detection of relativistic neutrons by $\mathrm{BaF}_{2}$ scintillators, Nucl. Instr. Meth. A394 (1997) 332-340

[98] S. Kubota et al., Response of $\mathrm{BaF}_{2}, \mathrm{BaF}_{2}$-plastic and $\mathrm{BGO}$ scintillators to neutrons with energies between 15 and $45 \mathrm{MeV}$, Nucl. Instr. Meth. A285 (1989) 436-440

[99] V.V. Avdeichikov et al, Light output and energy resolution of CSI, YAG, GSO, BGO and LSO scintillators for light ions, Nucl. Instr. Meth. A349 (1994) 216-224

[100] M.J. Weber, Scintillation mechanisms and new crystals, Nucl. Instr. Meth. A527 (2004) 9-14

[101] M. Kobayashi et al., Improvement in radiation hardness of PbWO4 scintillating crystals by La-doping, Nucl. Instr. Meth. A404 (1998) 149-156

[102] M. Kobayashi, Y. Usuki, M. Ishii, M. Itoh, M. Nikl, Further study on different dopings into PbWO4 single crystals to increase the scintillation light yield, Nucl. Instr. Meth. A540 (2005) 381-394

[103] P. Lecoq, Ten years of lead tungstate development, Nucl. Instr. Meth. A537 (2005) 15-21

[104] J.B. Birks, Scintillations from organic crystals: specific fluorescence and relative response to different radiations, Proc. Phys. Soc. A64 (1951) 874-877

[105] C.N. Chou, The nature of the saturation effect of fluorescent scintillators, Phys. Rev. 87 (1952) 904-905

[106] G.T. Wright, Scintillation response of organic phosphors, Phys. Rev. 91 (1953) 1282-1283

[107] R. Voltz, J. Lopes da Silva, G. Laustriat, A. Coche, Influence of the nature of ionising particles on the specific luminescence of organic scintillators, J. Chem. Phys. 45 (1966) 33063311

[108] R. Voltz, G. Laustriat, Radioluminescence des Milieux organiques I. Etude cinetique, J. Physique 29 (1968) 159-166

[109] R. Voltz, H. DuPont, G. Laustriat, Radioluminescence des Milieux organiques II. Verification experimentale de l'Etude cinetique, J. Physique 29 (1968) 297-305

[110] T.A. King, R. Voltz, The time dependence of scintillation intensity in aromatic materials, Proc. Roy. Soc. A (Lond.) 289 (1966) 424-439

[111] J. Lopes da Silva, R. Voltz, Variation du rendement de radioluminescence des scintillateurs organiques avec la perte d'energie et le nombre de charges des particules ionisantes, Rev. de Physique Appl. 7 (1972) 127-132

[112] R.L. Craun, D.L. Smith, Analysis of response data for several organic scintillators, Nucl. Instr. Meth. 80 (1970) 239-244

[113] D.L. Smith, R.G. Polk, T.G. Miller, Measurement of the response of several organic scintillators to electrons, protons and deuterons, Nucl. Instr. Meth. 64 (1968) 157-166

[114] V.V. Verbinski, W.R. Burrus, T.A. Love, W. Zobel, N.W. Hill, R. Textor, Calibration of an organic scintillator for neutron spectrometry, Nucl. Instr. Meth. 65 (1968) 8-25 
[115] G. Dietze, H. Klein, Gamma-calibration of NE213 scintillation counters, Nucl. Instr. Meth. 193 (1982) 549-556

[116] G. Dietze, Energy calibration of NE213 scintillation counter by $\gamma$-rays, IEEE-Trans. NS-26 (1979) 398-402

[117] L. Büermann, S. Ding, S. Guldbakke, H. Klein, T. Novotny, M. Tichy, Response of NE213 liquid scintillation detectors to high-energy photons (E $>3 \mathrm{MeV})$, Nucl. Instr. Meth. A332 (1993) 483-492

[118] T. Novotny, L. Büermann, S. Guldbakke, H. Klein, Response of NE213 liquid scintillation detectors to high-energy photons $\left(7 \mathrm{MeV}<E_{\gamma}<20 \mathrm{MeV}\right)$, Nucl. Instr. Meth. A400 (1997) 356366

[119] T. Novotny, Photon spectrometry in mixed neutron-photon fields using NE213 liquid scintillation detectors, PTB-N-28, Braunschweig, 1997

[120] A. Zimbal, H. Klein, M. Reginatto et al, High-resolution spectrometry with liquid scintillation detectors for fusion applications, This Workshop (2006) paper OE06

[121] M. Drosg, P.M. Drake, P. Lisowski, The contribution of carbon interactions to the neutron counting efficiency of organic scintillators, Nucl. Instr. Meth. 176 (1980) 477- 480

[122] E. Dekempeneer, H. Liskien, L. Mewissen, F. Portmans, A spectrometer for doubledifferential neutron-emission cross section measurements in the energy range 1.6 to $16 \mathrm{MeV}$, Nucl. Instr. Meth. A256 (1987) 489-498

[123] H. Klein, H. Schölermann, Improvement of the light collection in scintillation detectors, IEEETrans. NS-26 (1979) 373-377

[124] H. Schölermann, H. Klein, Optimising the energy resolution of scintillation counters at high energies, Nucl. Instr. Meth. 169 (1980) 25-31

[125] E. Frlez, B.K. Wright, D. Pocancic, optics: general-purpose scintillator light response simulation code, Comp. Phys. Comm. 134 (2001) 110-135

[126] N. Ghal-Eh, M.C. Scott, R. Koohi-Fayegh, M.F. Rahimi, A photon transport model code for use in scintillation detectors, Nucl. Instr. Meth. A516 (2004) 116-121

[127] G.W. McKinney, Review of Monte-Carlo all-particle transport codes and overview of recent MCNPX features, This Workshop (2006) paper RC01

[128] G. Dietze, H. Klein, NRESP4 and NEFF4 - Monte Carlo codes for the calculation of neutron response functions and detection efficiencies for NE213 scintillation detectors, PTB-ND-22, Braunschweig, 1982

[129] J.K. Dickens, SCINFUL: A Monte Carlo based computer program to determine a scintillator full energy response to neutron detection for En between $0.1 \mathrm{MeV}$ and $80 \mathrm{MeV}$, Reports ORNL-6462 (part 1) and ORNL-6463 (part 2), Oak Ridge, 1988

[130] M. Drosg, Accurate measurement of the counting efficiency of an NE213 neutron detector between 2 and $26 \mathrm{MeV}$, Nucl. Instr. Meth. 105 (1972) 573-584

[131] D. Schmidt, H. Klein, Precise time-of-flight spectrometry of fast neutrons: principles, methods and results, PTB-N-35, Braunschweig, 1998

[132] J. Cub, E. Finckh, K. Gebhardt, K. Geißdörfer, R. Lin, J. Strate, H. Klein, The neutron detection efficiency of NE213 detectors measured by means of a Cf-source, Nucl. Instr. Meth. A274 (1989) 217-221

[133] D. Schmidt, R. Böttger, Absolute determination of neutron detection efficiencies of NE213 detectors using a Cf-252 source in tof-measurements, PTB-N-42, Braunschweig, 2001

[134] F.D. Brooks, Scintillation counters with pulse shape selection to distinguish neutrons from gamma-rays, in: Liquid scintillation counting (1958) 268-269, (ed. C.G. Bell, F.N. Hayes, Pergamon, New York)

[135] F.D. Brooks, A scintillation counter with neutron and gamma-ray discriminators, Nucl. Instr. Meth. 4 (1959) 151-163

[136] L.M. Bollinger, G.E. Thomas, Measurement of the time dependence of scintillation intensity by a delayed-coincidence method, Rev. Sci. Instr. 32 (1961) 1044-1050

[137] F.T. Kuchnir, F.J. Lynch, Time dependence of scintillations and the effect on pulse-shape discrimination, IEEE NS-15 (1968) 107-113 
[138] L.J. Perkins, M.C. Scott, The application of pulse shape discrimination in NE213 to neutron spectrometry, Nucl. Instr. Meth. 166 (1979) 451-464

[139] F.W.K. Firk, Recent developments in neutron detection, in Fast Neutron Physics Part 2 (Ed. J.B. Marion \& J.F. Fowler), Interscience, New York, 1960, p 2237-2248

[140] N.V. Kornilov et al., Neutron spectroscopy with fast waveform digitizer, Nucl. Instr. Meth. A497 (2003) 467-478

[141] J.M. Adams, G. White, A versatile shape discriminator for charged particle separation and its application to fast neutron time-of-flight spectroscopy, Nucl. Instr. Meth. 156 (1978) 459-476

[142] P. Sperr, H. Spieler, M.R. Maier, D. Evers, A simple pulse-shape discrimination circuit, Nucl. Instr. Meth. 116 (1974) 55-59

[143] C.L. Morris, J.E. Bolger, G.W. Hoffmann, C.F. Moore, L.E. Smith, H.A. Thiessen, A digital technique for neutron-gamma pulse shape discrimination, Nucl. Instr. Meth. 137 (1976) 397398

[144] J.R.M Annand, A fast module for pulse shape analysis, Nucl. Instr. Meth. A262 (1987) 371377

[145] J. Bialkowski, M. Moszynski, D. Wolski, A pulse shape discriminator with high precision of neutron and gamma-ray selection at high counting rate, Nucl. Instr. Meth. A275 (1989) 322328

[146] M. Moszynski, G.J. Costa, G. Guillaume, B. Heusch, A. Huck, S. Mouatassim, Study of $n-\gamma$ discrimination with NE213 and BC501A liquid scintillators, Nucl. Instr. Meth. A350 (1994) 226-234

[147] O. Barnaba, Y.B. Chen, G. Musitelli, R. Nardo, G.L. Raselli, M. Rosella, P. Torre, A fullintegrated pulse-shape discriminator for liquid scintillator counters, Nucl. Instr. Meth. A410 (1998) 220-228

[148] Y. Kaschuk, B. Esposito, Neutron/ $\gamma$-ray digital pulse shape discrimination with organic scintillators, Nucl. Instr. Meth. A551 (2005) 420-428

[149] B. Esposito, A Zimbal et al, An FPGA-based digital system for the acquisition of neutron pulse height spectra with liquid scintillation detectors, This Workshop (2006) paper OD01

[150] L. Lebreton, J.F. Guerre-Chaley et al, Performances of digital acquisition for a BC501A detector system, This Workshop (2006) paper OD02

[151] Z.W. Bell, Tests on a digital neutron-gamma pulse shape discriminator with NE213, Nucl. Instr. Meth. 188 (1981) 105-109

[152] F.-J Hambsch, Digitisation techniques applied in nuclear experiments, This Workshop (2006) paper RD01

[153] M. Reginatto, A. Zimbal, Bayesian methods for the analysis of pulse height spectra from liquid scintillator detectors, This Workshop (2006) paper OD03

[154] M. Reginatto, P. Goldhagen, S. Neumann, Spectrum unfolding, sensitivity anlysis and propagation of uncertainties with the maximum entropy deconvolution code MAXED, Nucl. Instr. Meth. A476 (2002) 242-246

[155] M. Matzke, Unfolding procedures, Radiat. Prot. Dosim. 107 (2003) 111-124

[156] H. Klein, D. Thomas, H.G. Menzel, G. Curzio, F. d'Errico Eds., NEUSPEC 2000, Proceedings of the International Workshop on Neutron Field Spectrometry in Science, Technology and Radiation Protection, Pisa, Italy, June 4-8, 2000, Nucl. Instr. Meth. A476 (2000)

[157] D.J. Thomas, H. Klein, Eds., Handbook on Neutron and Photon Spectrometry Techniques for Radiation Protection, Radiat.. Prot. Dosim. 107 (2003)

[158] C. Renner, N.W. Hill, G.L. Morgan, K. Rush, J.A. Harvey, Absolute neutron flux measurements using an NE110 scintillation counter, Nucl. Instr. Meth. 154 (1978) 525-533

[159] R.E. Textor, V.V. Verbinski, O5S, a Monte Carlo code for calculating pulse height distributions due to monoenergetic neutrons incident on organic scintillators, ORNL-4160, Oak Ridge, 1968

[160] W.P. Pönitz, The black neutron detector, Nucl. Instr. Meth. 109 (1973) 413-420

[161] K.C. Duvall, A.D. Carlson, O.A. Wasson, Calibration of the NBS black detector at $2.3 \mathrm{MeV}$ using the time-correlated associated-particle method, Nucl. Instr. Meth. B10/11 (1985) 931933 
[162] M.S. Dias, R.G. Johnson, O.A. Wasson, Design and calibration of an absolute flux detector for 1-15 MeV neutrons, Nucl. Instr. Meth. 224 (1984) 532-546

[163] J.B. Czirr et al., Capture-gated neutron spectrometry, Nucl. Instr. Meth. A476 (2002) 309-312

[164] M. Hayashi, Y. Watanabe et al, Fast response of a ${ }^{6}$ Li-loaded liquid scintillator, This Workshop (2006) paper PA07

[165] M. Takada et al., Characteristics of a phoswich detector to measure the neutron spectrum in a mixed field of neutrons and charged particles, Nucl. Instr. Meth. A476 (2002) 332-336

[166] K. Gunzert-Marx, D. Schardt et al, Response of a barium-fluoride scintillation detector to fast neutrons in the range of 45 to $198 \mathrm{MeV}$, This Workshop (2006) paper PA07

[167] K. Gunzert-Marx, G. Fehrenbacher et al, Double differential neutron yields from thick targets induced by relativistic carbon and uranium beams, This Workshop (2006) paper PA07

[168] M.S. Derzon, D.R. Slaughter, S.G. Prussin, A high-pressure ${ }^{3}$ He gas scintillation spectrometer, IEEE Trans. Nucl. Sci. NS 33 (1986) 247-249

[169] D. Vartsky, Prospects of fast-neutron resonance radiography and requirements for instrumentation, This Workshop (2006) paper RA02

[170] V. Dangendorf, C. Kersten, G. Laczko, D. Vartsky, I. Mor, M.B. Goldberg, G. Feldman, A. Breskin, R. Chechik, O. Jagutzky, U. Spillman, Detectors for energy-resolved fast neutronimaging, Nucl. Instr. Meth. A535 (2004) 93-97

[171] D. Vartsky, I. Mor, M.B. Goldberg, I. Mardor, G. Feldman, D. Bar, A. Shor, V. Dangendorf, G. Laczko, A. Breskin, R. Chechik, Time-resolved fast neutron imaging: simulation of detector performance, Nucl. Instr. Meth. A542 (2005) 206-212

[172] G. Bonheure, Neutron diagnostics for reactor scale fusion experiments: a review of JET systems, This Workshop (2006) paper RE02

[173] W.L. Reiter, G. Stengl, A blue light emitting diode used as a reference element in scintillation spectrometers, Nucl. Instr. Meth. 180 (1981) 105-107

[174] Y. Holler, J. Koch, A. Naini, A stabilised NE213 scintillator for neutron tof-spectroscopy, Nucl. Instr. Meth. 204 (1983) 485-490

[175] L. Holm, H.W. Fielding, G.C. Neilson, Gain stabilisation of phototubes using an LED diode scheme, Nucl. Instr. Meth. A234 (1985) 517-520

[176] P. Hanlet et al, DO Myon scintillation counters, Nucl. Instr. Meth. A521 (2004) 343-360

[177] D. Renker, Photosensors, Nucl. Instr. Meth. A527 (2004) 15-20

[178] C.L. Melcher, Perspectives on the future development of new scintillators, Nucl. Instr. Meth. A537 (2005) 6-14 